\title{
Electric field effects on the ionic-neutral curve crossing of alkali halide molecules
}

\author{
Carmen Sousa, David Domínguez-Ariza, Coen de Graaf, and Francesc Illas \\ Departament de Química Física i Centre Especial de Recerca en Química Teòrica, \\ Universitat de Barcelona, Martí i Franquès 1, 08028 Barcelona, Spain
}

(Received 6 June 2000; accepted 15 September 2000)

\begin{abstract}
The weakly avoided crossing between the two lowest ${ }^{1} \Sigma^{+}$electronic states of a series of alkali halide molecules has been studied by means of the recently reported multistate complete active space second-order perturbation theory, MS-CASPT2, method. For a large enough basis set and a complete active space self-consistent field that includes part of the radial and angular correlation of the outermost halide electrons, the calculated crossing distance is in very good agreement with that predicted from the Rittner empirical potential. The study of the relevant parameters corresponding to the crossing region on these molecules has been extended to include the effect of a uniform electric field and a generalization of the empirical Rittner formula that includes the electric field effects is presented. The predictions made by the MS-CASPT2 method are also in agreement with those derived from the generalized Rittner potential. Finally, the possible implications of the present work on electron transfer processes at metal electrodes are discussed. (C) 2000 American Institute
\end{abstract} of Physics. [S0021-9606(00)30846-7]

\section{INTRODUCTION}

The chemical bond in alkali halide diatomic molecules prompts a marked ionic character in their electronic ground state wave function at the equilibrium geometry. This is in contrast with the situation for the atoms at infinite separation where the electronic ground state does not exhibit any charge separation. A simple quantum chemical picture of this phenomenon can be obtained from valence bond theory. Near the equilibrium geometry the ground state wave function of these molecules is dominated by the $\mathrm{M}^{+}-\mathrm{X}^{-}$ionic components with a small contribution of the neutral $\mathrm{M}-\mathrm{X}$ valence bond determinants whereas at long distances the situation is the opposite. Therefore, the diabatic curves of the two lowest ${ }^{1} \Sigma^{+}$states of the alkali halide molecules must undergo a crossing, probably at large distances. In the adiabatic representation the identical symmetry of both electronic states results in an avoided crossing. Since the interaction between the ionic and neutral state at the crossing point is in general rather small the crossing is often weakly avoided. ${ }^{1}$ This situation is not exclusive of molecular systems, it is also encountered for different kinds of adsorbates on metal surfaces when the interaction occurs through an ionic bond. ${ }^{2,3} \mathrm{Ex}-$ amples of ionic adsorption concern halides on $\mathrm{Cu}(100){ }^{4}$ $\operatorname{Ag}(111){ }^{5}$ and on $\mathrm{Hg}$ surfaces, ${ }^{6}$ alkali metals on metal surfaces, ${ }^{7-10}$ and $\mathrm{NO}$ on $\mathrm{Cu}(111)^{2}$ and $\mathrm{Ag}(111)^{11}$ surfaces. The existence of the neutral-ionic crossing has consequences on the dynamics of the gas-surface reaction, ${ }^{2,3}$ and for this reason, the neutral-ionic crossing is at the hearth of oxidation-reduction processes at metal surfaces and is relevant to both electrochemistry and corrosion science. Understanding the charge-transfer process between an adsorbate and a metal surface at a molecular level requires a powerful methodology capable of properly describing the crossing region and, eventually, of its dependence on external potentials or electric fields. However, the neutral-ionic crossing is a rather complicated process and its quantitative study at a quantum mechanical level requires the use of sophisticated state-of-the-art computational methods not available until present days (vide infra).

For diatomic molecules, the crossing distance of the ionic and neutral curves, $R_{c}$, has been estimated ${ }^{12,13}$ using the electrostatic Rittner potential for the ionic state. In this way $R_{c}$ can be written as a function of empirical parameters as the ionization potential of the alkali atom, IP(M), the electron affinity of the halogen, $\mathrm{EA}(\mathrm{X})$, and the polarizabilities $(\alpha)$ of both ions, $\mathrm{M}^{+}$and $\mathrm{X}^{-}$. The following iterative expression was derived:

$$
R_{c}=\frac{1}{\Delta E_{\infty}}+\frac{\alpha\left(\mathrm{M}^{+}\right)+\alpha\left(\mathrm{X}^{-}\right)}{2 \Delta E_{\infty} R_{c}^{3}}
$$

with $\Delta E_{\infty}$ being the energy difference of the neutral and ionic asymptotes at infinite internuclear distance, i.e., $\mathrm{IP}(\mathrm{M})-\mathrm{EA}(\mathrm{X})$. Since the crossing occurs at rather large distances, a good estimation of $R_{c}$ can be obtained by neglecting the contribution arising from the polarizability of both ions, i.e., ignore the second term in Eq. (1). The interest in the crossing of these two diabatic curves is not limited to the precise location of the crossing point, the energy difference between the adiabatic curves at this point is also relevant because it corresponds to twice the diabatic coupling element. In most cases the neutral-ionic crossing results in a potential energy barrier-e.g., the adsorption of $\mathrm{NO}$ on metal surfaces $^{2,3}$ - and the diabatic coupling term plays a crucial role in determining the exact barrier height, and hence, the kinetics of the whole process. According to the semiempirical expression derived by Grice and Herschbach, ${ }^{14}$ the electronic coupling term decreases exponentially with $R_{c}$. 
Both the position of the avoided crossing and the magnitude of the coupling are very sensitive to the energy difference between the two adiabatic curves and any bias on the treatment of one of the two states leads to large changes in both properties. For this reason, the weakly avoided crossing problem has been a challenge for any methodological treatment. Werner and Meyer ${ }^{12}$ performed multiconfigurational self-consistent field, MCSCF, calculations for $\mathrm{LiF}$ and their results confirm the predictions made by the empirical expressions for $R_{c}$ and the electronic coupling. These authors point out the importance of properly describing the potential energy curve asymptotic limit, IP(M)-EA(X), to obtain a reasonable value of $R_{c}$. Bauschlicher and Langhoff ${ }^{15}$ investigated the reliability of different theoretical approaches to predict the correct value of the crossing point of the $\mathrm{LiF}$ molecule. These authors compared the potential energy and dipole moment curves obtained by means of full configuration interaction, FCI, and multireference configuration interaction, MRCI, calculations, based on a complete active space self-consistent field, CASSCF, reference wave function. Bauschlicher and Langhoff ${ }^{15}$ concluded that reaching a correct description of the avoided crossing region requires the use of molecular orbitals optimized in a state-average CASSCF calculation with equal weights for the ionic and neutral solutions.

In some systems state-average MCSCF potential energy surfaces can present discontinuities ${ }^{16}$ and, hence, this approach is not the most appropriate choice to investigate the crossing region. However, for the present purposes this is an adequate method since no discontinuities are found for LiF. ${ }^{15}$ Nevertheless, state-average CASSCF, hereafter referred to as SA-CASSCF, potential curves considerably underestimate the crossing point distance. This is due to the lack of dynamical correlation, which is larger for the ionic than for the neutral state. Including the pertinent electronic correlation effects is not a simple task and the usual second-order perturbation theory based approaches break down. Malrieu et al. ${ }^{17}$ have demonstrated that the CASPT $2^{18}$ second-order perturbation theory method that uses a CASSCF as zerothorder wave function leads to energy curves for the ionic and neutral states of $\mathrm{LiF}$ that cross at two points, $\sim 4.8$ and $\sim 5.6$ $\AA$. To properly describe the avoided crossing region, various methodological improvements have been developed in the last few years. García et al. ${ }^{19,20}$ designed a CI method focussed on the calculation of energy differences in which the orbital set is iteratively constructed, the so-called iterative difference-dedicated CI, IDDCI, method. Using the same basis set as in the FCI calculations of Ref. 15, the authors found a single crossing point at $6.3 \AA$, comparable with the $6.67 \AA$ obtained in the MRCI calculations ${ }^{15}$ but still far from the $7.24 \AA$ value predicted from the Rittner potential, cf. Eq. (1). More recently, an extension of the CASPT2 method has been developed, in which several electronic states can be coupled at second order via an effective Hamiltonian approach. ${ }^{21}$ This new methodology, denoted as multistate CASPT2, or more briefly MS-CASPT2, has been applied to the description of the avoided crossing in LiF. The MSCASPT2 potential energy curves exhibit the correct behavior in the avoided crossing region ${ }^{22}$ and good agreement with previous results. ${ }^{15}$ Similar findings were also obtained by Nakano $^{23}$ based on second-order quasidegenerate perturbation theory.

The interest in the avoided crossing of these kind of molecules goes well beyond the methodological point of view. In fact, femtosecond real-time dynamics experiments have been reported for $\mathrm{NaI},{ }^{24,25} \mathrm{NaBr}$, and LiI. ${ }^{26}$ From these experiments it is possible to obtain direct information about the electronic coupling term and on the location of the crossing point. Theoretical studies for these molecules, mainly based on MRCI wave functions, have also been reported in the last few years. ${ }^{27-29}$ Despite the drawbacks of the MRCI wave function to properly describe the avoided crossing region above commented, a rather good agreement between MRCI results and experiment is found for these molecules. This is due to a fortuitous error cancellation between the underestimation of halide EA and the neglect of spin-orbit interaction.

A methodology able to accurately describe ground and excited adiabatic potential energy curves in the whole interval of internuclear distances permits an accurate description of spectroscopic parameters of the low-lying states of diatomic molecules but also of the possible avoided crosses as those described above. Moreover, the impact of such a methodology goes beyond the study of diatomic molecules as it will permit to characterize electron-transfer reactions either between different species or between an adsorbate and a surface. As pointed out above, the potentiality to study chargetransfer reactions at a surface may have a large impact on understanding electrochemical processes at a quantummechanical level. This possibility acted precisely as the catalyst that triggered the present study. In this paper we apply the MS-CASPT2 approach to quantitatively describe the avoided crossing region on a family of alkali halide molecules, $\mathrm{MX}$ with $\mathrm{M}=\mathrm{Li}, \mathrm{Na}$ and $\mathrm{X}=\mathrm{F}, \mathrm{Cl}$, to get insight into the value of the crossing distance and of the electronic coupling at this point. It is worth pointing out that the diabatic coupling element, $H_{12}$, matches half the adiabatic energy difference at the crossing point only. To provide the values of the electronic coupling terms as a function of the distance, diabatic curves have also been derived. In addition, an attempt is made to relate the neutral-ionic avoided crossing with charge-transfer electrochemical processes. To this end, the variation of the crossing distance, $R_{c}$, and of the electronic coupling term with respect to an external uniform electric field will be examined along the alkali halide series. The field is oriented in the molecular axis with the metal placed at the origin and the halogen on the positive part of the $z$ axis. The results obtained at zero electric field are compared with those extracted from the empirical formula [Eq. (1), first right-hand side term only]. An empirical equation for $R_{c}$ in the presence of a uniform external field has been derived using the same physical reasoning employed to obtain Eq. (1). Neglecting the terms that include the polarizability of the ions, which at the distances where the crossing occurs are expected to be small, one obtains

$$
R_{c}=\frac{\Delta E_{\infty} \pm \sqrt{\Delta E_{\infty}^{2}-4 q^{3} F}}{2 q F}
$$


with $F$ being the amplitude of the uniform electric field and $q$ the absolute value of the atomic charge in the ionic configuration, i.e., 1 for the alkali halides studied here. A positive external field stabilizes the ionic asymptote and two possible values of $R_{c}$ exist. The first one corresponds to the crossing in which the ground state changes its character from ionic to neutral. The second crossing, which has no further interest, occurs at larger distances because of the stabilization of the ionic curve by the electric field. For intense electric fields the ionic configuration stabilizes so much that crossing does not occur at all. The maximum value of the electric field for which a curve crossing is still observed is

$$
F=\frac{\Delta E_{\infty}^{2}}{4 q^{3}} .
$$

For negative electric fields, Eq. (2) gives only one meaningful solution, the one with a positive value of $R_{c}$. In this case, the ionic configuration is destabilized and the crossing happens at shorter distances compared to the unperturbed system.

\section{COMPUTATIONAL DETAILS}

At the equilibrium distance the $X^{1} \Sigma^{+}$ground state of the alkali halide molecules is well described by a [core] $1 \sigma^{2} 2 \sigma^{2} 1 \pi^{4}$ configuration state function that has essentially an ionic character. Here, the $1 \sigma, 2 \sigma, 3 \sigma$, and $1 \pi$ molecular orbitals refer to the orbitals coming from the combination of the valence alkali $s$ orbital $(2 s$ for $\mathrm{Li}$ and $3 s$ for $\mathrm{Na}$ ) and the $s$ and $p$ valence orbitals of the halogen atoms $(2 s, 2 p$ for $\mathrm{F}$ and $3 s, 3 p$ for $\mathrm{Cl}$ ). For the first ${ }^{1} \Sigma^{+}$excited state, the neutral configuration, [core] $1 \sigma^{2} 2 \sigma^{1} 3 \sigma^{1} 1 \pi^{4}$ dominates the electronic wave function. State average CASSCF wave functions for the two lowest ${ }^{1} \Sigma^{+}$states of all molecules are computed and used as zeroth-order reference wave functions in a subsequent MS-CASPT2 (Ref. 21) calculation. The SACASSCF wave functions are coupled at second order via an effective $(2 \times 2)$ Hamiltonian in which the diagonal elements are equal to the single-state CASPT2, SS-CASPT2, energies. All calculations have been performed using the MOLCAS4 software package. ${ }^{30}$

Since the description of the avoided crossing region may be affected by the particular choice of the one-electron basis sets and by the definition of the active space, a systematic study is indispensable. To study the dependence of the results on the one-electron basis set and on the active space used to construct the CASSCF wave functions, we consider basis sets of different quality and active spaces of various sizes. For $\mathrm{Li}$ and $\mathrm{F}$ a $(14 s 9 p 4 d 3 f)$ primitive basis set is contracted to three different ANO basis sets: $[3 s 2 p 1 d]$, $[4 s 3 p 2 d 1 f]$, and $[5 s 4 p 3 d 2 f] .{ }^{31}$ For $\mathrm{Na}$ and $\mathrm{Cl}$ the primitive sets include $(17 s 12 p 5 d 4 f)$ functions and is contracted to $[4 s 3 p 2 d 1 f], \quad[5 s 4 p 3 d 2 f]$, and $[6 s 5 p 4 d 3 f] .{ }^{32}$ Three different SA-CASSCF wave functions have been constructed. For the simplest one, the active space contains two orbitals, $2 \sigma$ and $3 \sigma$, and two electrons. These calculations are referred to as CASSCF (2000), the numbers in parentheses indicate the number of active orbitals on each irreducible symmetry representation of the $C_{2 v}$ point
TABLE I. Dependence of the crossing distance $\left(R_{c}\right.$ in $\AA$ ) and of the asymptotic energy difference $\left(\Delta E_{\infty}\right.$ in $\left.\mathrm{eV}\right)$ on the basis set and the size of the complete active space. Results for the LiF molecule.

\begin{tabular}{|c|c|c|c|c|c|c|}
\hline \multirow[b]{2}{*}{ Basis } & \multicolumn{2}{|c|}{$\left[\begin{array}{lll}3 s & 2 p & 1 d\end{array}\right]$} & \multicolumn{2}{|c|}{$\left[\begin{array}{llll}4 s & 3 p & 2 d & 1 f\end{array}\right]$} & \multicolumn{2}{|c|}{$\left[\begin{array}{lllll}5 s & 4 p & 3 d & 2 f\end{array}\right]$} \\
\hline & $\begin{array}{l}R_{c} \\
(\AA)\end{array}$ & $\begin{array}{l}\Delta E_{\infty} \\
(\mathrm{eV})\end{array}$ & $\begin{array}{l}R_{c} \\
(\AA)\end{array}$ & $\begin{array}{l}\Delta E_{\infty} \\
(\mathrm{eV})\end{array}$ & $\begin{array}{l}R_{c} \\
(\AA)\end{array}$ & $\begin{array}{l}\Delta E_{\infty} \\
(\mathrm{eV})\end{array}$ \\
\hline CASSCF (2000) & 4.34 & 3.72 & 4.26 & 3.80 & 4.25 & 3.82 \\
\hline$+\mathrm{PT} 2$ & 5.65 & 2.69 & 6.86 & 2.25 & 7.32 & 2.03 \\
\hline CASSCF (2220) & 4.53 & 3.50 & 4.56 & 3.46 & 4.57 & 3.45 \\
\hline$+\mathrm{PT} 2$ & 5.88 & 2.55 & 6.70 & 2.26 & 6.83 & 2.16 \\
\hline CASSCF (5331) & 6.40 & 2.25 & 6.50 & 2.24 & 6.50 & 2.23 \\
\hline$+\mathrm{PT} 2$ & 6.11 & 2.41 & 6.60 & 2.22 & 6.78 & 2.17 \\
\hline
\end{tabular}

group symmetry $\left(a_{1}, b_{1}, b_{2}, a_{2}\right)$. In order to obtain a more accurate description of the halogen electron affinity, it is convenient to include most of the $n p$ to $(n+1) p$ radial correlation of the halide atom in the CAS wave function, with $n=2$ for $\mathrm{F}$ and $n=3$ for $\mathrm{Cl}$. Following previous work, ${ }^{15}$ we therefore first extend the active space to (2220) with the $1 \pi$ orbitals $-b_{1}$ and $b_{2}$ in the $C_{2 v}$ point group-and an additional set of virtual orbitals of the same symmetries. Finally, the CAS is expanded to (5331) adding the five components of the halogen $3 d$ shell and the virtual halogen $p \sigma$ orbitals. The inclusion of the halogen $3 d$ orbitals in the active space permits to variationally account for part of the angular correlation of the $n p$ electrons and, hence, has a direct effect on the calculated halide EA. Likewise, the inclusion of the halogen $p \sigma$ virtual orbitals permits a better description of the radial correlation of the halogen $n p$ electrons: notice that only radial correlation in the $\pi$ space is taken into account in the (2220) space. In the subsequent second-order perturbation calculations, all electrons except the deep-core electrons are correlated, i.e., the $\mathrm{Li}, \mathrm{Na}$, and $\mathrm{F} 1 s^{2}$ electrons and the Cl $1 s^{2} 2 s^{2} 2 p^{6}$ electrons are excluded.

\section{RESULTS AND DISCUSSION}

\section{A. The effect of active space and the one-electron basis set}

Table I shows the crossing distances and the asymptotic energy differences, $\Delta E_{\infty}$, of the two lowest ${ }^{1} \Sigma^{+}$states of LiF computed using different basis sets and CAS spaces. As expected, we observe that for a given active space the CASSCF crossing point and $\Delta E_{\infty}$ do not change with the basis set used. The dependence on the size of the active space, however, is more pronounced. CASSCF calculations with (2000) and (2220) active spaces underestimate the electron affinity of $\mathrm{F}$ resulting in too large a $\Delta E_{\infty}$ and in too short an estimate of the crossing point distance, in agreement with the prediction of Eq. (1). Increasing the active space with the fluorine $3 d$ virtual orbitals largely improves the calculated EA and the crossing point becomes $6.5 \AA$, about $2 \AA$ longer than for the smaller active spaces. For the other molecules, the effect of extending the CAS with the halogen $3 d$ orbitals is even larger.

On the other hand, CASPT2 results show dependence on both the CAS size and on the basis set used. For a given CAS, an increase of the calculated $R_{c}$ value on increasing the 
TABLE II. Calculated CASPT2-based on a SA-CASSCF (5331) zeroth-order wave function-and experimental (in parentheses) spectroscopic constants of the ${ }^{1} \Sigma^{+}$ground state of the alkali halide molecules. Values are reported for the internuclear equilibrium distance, $R_{e}$, vibrational frequency, $\omega_{e}$, rotational constant, $B_{e}$, vibration-rotational coupling, $\alpha_{e}$, centrifugal distortion constant, $D$, dipole moment, $\mu_{e}$, and dissociation energy, $D_{e}$.

\begin{tabular}{lccccccc}
\hline \hline & $R_{e}(\AA)$ & $\omega_{e}\left(\mathrm{~cm}^{-1}\right)$ & $B_{e}\left(\mathrm{~cm}^{-1}\right)$ & $\alpha_{e}\left(10^{-3} \mathrm{~cm}^{-1}\right)$ & $D\left(10^{-6} \mathrm{~cm}^{-1}\right)$ & $\mu_{e}(D)$ & $D_{e}(\mathrm{eV})$ \\
\hline $\mathrm{LiF}$ & $\mathbf{1 . 5 6 5}(1.564)$ & $\mathbf{9 1 1}(910)$ & $\mathbf{1 . 3 4 4}(1.345)$ & $\mathbf{2 1 . 1}(20.3)$ & $\mathbf{1 1 . 8}(11.8)$ & $\mathbf{6 . 4 8 0}(6.325)$ & $\mathbf{5 . 9 5}(5.97)$ \\
$\mathrm{LiCl}$ & $\mathbf{2 . 0 3 4}(2.021)$ & $\mathbf{6 3 4}(643)$ & $\mathbf{0 . 6 9 7 3}(0.7065)$ & $\mathbf{7 . 7 6}(8.01)$ & $\mathbf{3 . 3 8}(3.41)$ & $\mathbf{7 . 3 6 7}(7.128)$ & $\mathbf{4 . 8 3}(4.88)$ \\
$\mathrm{NaF}$ & $\mathbf{1 . 8 9 9}(1.926)$ & $\mathbf{5 6 9}(536)$ & $\mathbf{0 . 4 4 9 6}(0.4369)$ & $\mathbf{5 . 0 9}(4.56)$ & $\mathbf{1 . 1 3}(1.16)$ & $\mathbf{8 . 2 1 6}(8.155)$ & $\mathbf{5 . 1 5}(5.36)$ \\
$\mathrm{NaCl}$ & $\mathbf{2 . 3 1 0}(2.361)$ & $\mathbf{3 8 7}(366)$ & $\mathbf{0 . 2 2 7 8}(0.2181)$ & $\mathbf{2 . 7 4}(1.62)$ & $\mathbf{0 . 3 2 1}(0.312)$ & $\mathbf{9 . 0 4 8}(9.118)$ & $\mathbf{4 . 3 1}(4.25)$ \\
\hline \hline
\end{tabular}

basis set quality is observed in all cases with a concomitant improvement of the calculated $\Delta E_{\infty}$ that approaches the 1.99 $\mathrm{eV}$ experimental value. The extension of the active space acts in the opposite way, $R_{c}$ becomes smaller going from CAS (2000) to CAS (2220), to CAS (5331), except for the smallest basis set. This indicates that a purely second-order perturbation treatment of the dynamical correlation effects leads to an overestimation of the position of the crossing point. This is repaired when the active space is augmented and the dynamical electron correlation effects are (in part) variationally treated. The combination of large active spaces and large one-electron basis sets lead to rather similar CASSCF and CASPT2 results. For LiF, results on Table I show that $R_{c}$ and $\Delta E_{\infty}$ are converged with a CASSCF (2220) calculation and the intermediate $[4 s 3 p 2 d 1 f]$ basis. The same behavior is observed for the $\mathrm{LiCl}$ and $\mathrm{NaF}$ molecules. However, for $\mathrm{NaCl}$ small but significant changes are observed when a larger basis set is applied and the active space is augmented to (5331). In order to be able to compare results between the different molecules, the largest basis set and the (5331) active space is applied throughout all calculations reported in this paper.

\section{B. Ground state spectroscopic constants}

The description of the two states of interest with an average set of orbitals may introduce uncontrolled inaccuracies in the calculations, especially in the region close to the equilibrium distance, where the energy separation between both states is quite large. To quantify this potential source of error we compare the experimental spectroscopic constants and dipole moment of the $X^{1} \Sigma^{+}$ground state for all four molecules, ${ }^{7} \mathrm{Li}^{19} \mathrm{~F},{ }^{7} \mathrm{Li}^{35} \mathrm{Cl},{ }^{23} \mathrm{Na}^{19} \mathrm{~F}$, and ${ }^{23} \mathrm{Na}^{35} \mathrm{Cl}$ with those ob- tained using the SA-CASSCF/CASPT2 method. The CASPT2 values reported in Table II have been obtained using a SA-CASSCF (5331) reference wave function constructed by averaging the two lowest ${ }^{1} \Sigma^{+}$states with the same weight. In addition, it is worth pointing out that the values obtained by a SS-CASSCF/CASPT2 calculation for the $X^{1} \Sigma^{+}$ground state (not reported) are virtually the same as those included in Table II. This fact shows that with such a large active space the effect of averaging the orbitals for two roots that are quite far away in energy does not seriously affect the calculated properties of the ground state that indeed compare very well with experiment. ${ }^{1}$

\section{Electric field effect on the parameters defining the crossing region}

The distance at which the crossing point between the ionic and the neutral states occurs and its variation with respect to a uniform external electric field have been studied for all four molecules. As commented above, the electric field is applied along the internuclear axis with the metal placed at the origin and the halogen on the $z$ axis in such a way that a positive electric field stabilizes the ionic state. To study the dependence of $R_{c}$ on the electric field, several fields of different intensity have been considered. Results are summarized in Table III, where the results without electric field are also given for comparison. For each molecule, three different values of $R_{c}$ are reported in Table III. Those are the MS-CASPT2 computed value; the value obtained by applying Eq. (2) [Eq. (1) for $\mathrm{F}=0$ ] with the MS-CASPT2 computed values for the halogen EA and metal IP; and, finally, the value derived from Eq. (2) using the experimental values for $\mathrm{EA}(\mathrm{X})$ and $\mathrm{IP}(\mathrm{M})$. First of all, notice that, as the electric

TABLE III. Crossing point (in $\AA$ ) as function of the applied homogeneous electric field ( $10^{-3}$ a.u.). For each molecule three entries are given. The first one (calc.) corresponds to the results of the MS-CASPT2 calculations, the second one [Eq. (2) (calc)] is obtained substituting the CASPT2 value of $\Delta E_{\infty}$ in Eq. (2). Finally, we list the results of Eq. (2) using the experimental $\Delta E_{\infty}$ [Eq. (2) (expt)].

\begin{tabular}{|c|c|c|c|c|c|c|c|c|c|c|c|c|}
\hline \multirow[b]{2}{*}{ Field } & \multicolumn{3}{|c|}{$\mathrm{LiF}$} & \multicolumn{3}{|c|}{$\mathrm{LiCl}$} & \multicolumn{3}{|c|}{$\mathrm{NaF}$} & \multicolumn{3}{|c|}{$\mathrm{NaCl}$} \\
\hline & Calc. & $\begin{array}{l}\text { Eq. (2) } \\
\text { (calc.) }\end{array}$ & $\begin{array}{c}\text { Eq. (2) } \\
\text { (expt.) }\end{array}$ & Calc. & $\begin{array}{l}\text { Eq. (2) } \\
\text { (calc.) }\end{array}$ & $\begin{array}{l}\text { Eq. (2) } \\
\text { (expt.) }\end{array}$ & Calc. & $\begin{array}{c}\text { Eq. (2) } \\
\text { (calc.) }\end{array}$ & $\begin{array}{l}\text { Eq. (2) } \\
\text { (expt.) }\end{array}$ & Calc. & $\begin{array}{l}\text { Eq. (2) } \\
\text { (calc.) }\end{array}$ & $\begin{array}{c}\text { Eq. (2) } \\
\text { (expt.) }\end{array}$ \\
\hline-0.50 & 6.33 & 6.18 & 6.66 & 7.25 & 7.09 & 7.35 & 7.31 & 7.09 & 7.45 & 8.34 & 8.26 & 8.31 \\
\hline 0.00 & 6.78 & 6.64 & 7.24 & 7.99 & 7.80 & 8.13 & 7.98 & 7.78 & 8.28 & 9.43 & 9.41 & 9.47 \\
\hline 0.50 & 7.38 & 7.26 & 8.08 & 8.99 & 8.88 & 9.43 & 9.06 & 8.88 & 9.65 & 11.62 & 11.72 & 11.85 \\
\hline 0.75 & 7.79 & 7.69 & 8.69 & 9.81 & 9.78 & 10.57 & 9.94 & 9.78 & 10.92 & 14.95 & 15.35 & 15.83 \\
\hline 1.00 & 8.37 & 8.25 & 9.61 & 11.29 & 11.39 & 13.19 & 11.31 & 11.39 & 14.42 & & & \\
\hline 1.15 & 8.80 & 8.70 & 10.50 & 13.62 & 14.55 & & 13.82 & 14.55 & & & & \\
\hline 1.50 & 10.85 & 10.72 & & & & & & & & & & \\
\hline
\end{tabular}




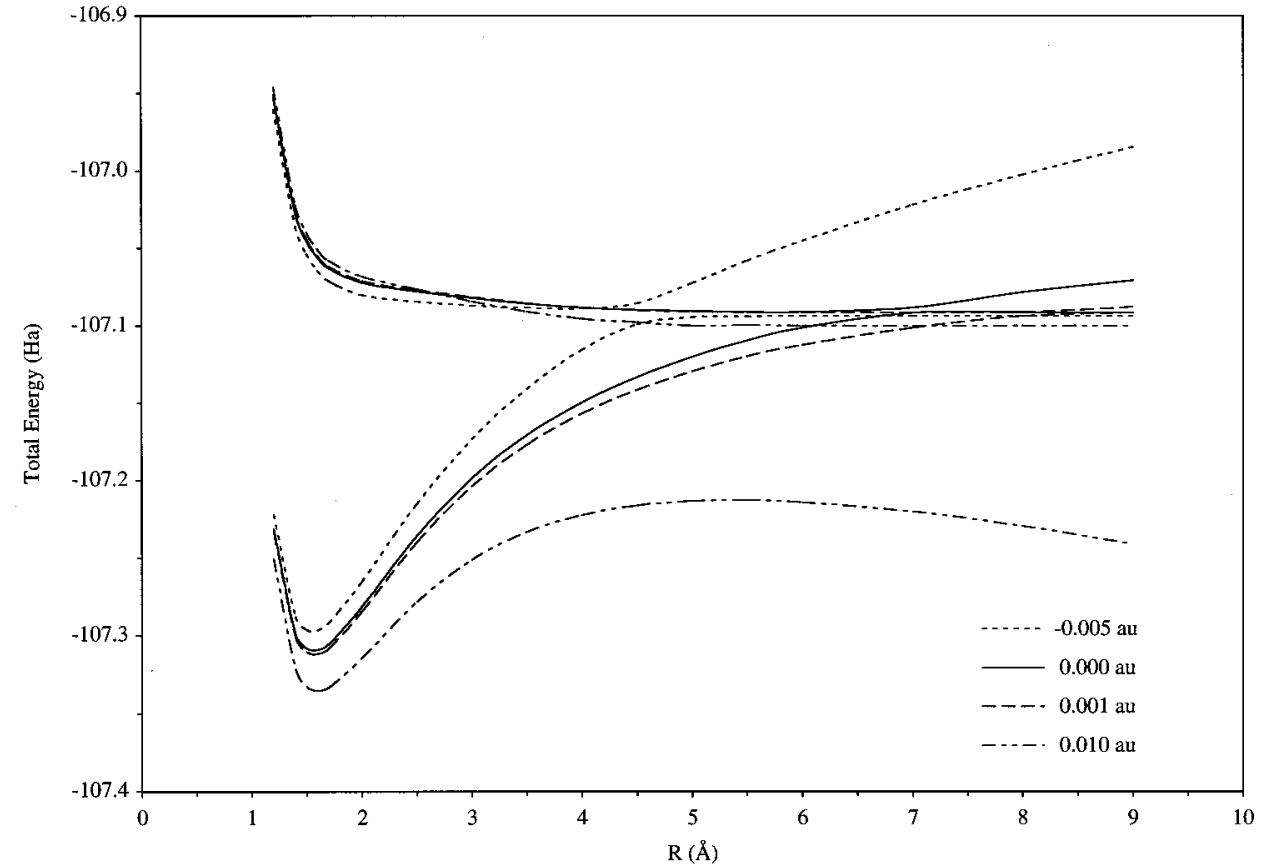

FIG. 1. MS-CASPT2 potential energy curves for $\mathrm{LiF}$ as a function of the intensity of a uniform external electric field in the direction of the internuclear axis. field strength increases, the crossing occurs at larger distance due to the stabilization of the ionic curve. On the contrary, for a negative electric field the ionic curve is destabilized and, therefore, the crossing distance decreases. This fact is illustrated in Fig. 1 for LiF; the same behavior is found for the rest of the molecules considered in the present study. In this figure, MS-CASPT2 potential energy curves for the two lowest ${ }^{1} \Sigma^{+}$states are plotted for various intensities of the external electric field including also the curves corresponding to $\mathrm{F}=0$. The close resemblance between the MSCASPT2 values reported in Table III and those derived from Eq. (1) and Eq. (2) (column 2 for each molecule) shows that the performance of the empirical formula is outstanding. This means that the crossing point is basically determined by the energy difference of the ionic and neutral curves at infinite internuclear distance, $\operatorname{IP}(\mathrm{M})-\mathrm{EA}(\mathrm{X})$, and by the intensity of the electric field applied. Moreover, results in Table III show that the effect of polarization terms, not included when only the first right-hand side term of Eq. (1) is considered and also neglected in the present derivation of Eq. (2) is very small and therefore can be neglected in both cases. Consequently, to properly predict the distance at which the crossing takes place it is strictly necessary to accurately compute the halogen EA and the alkali atom IP.

The experimental value of $\operatorname{IP}(\mathrm{M})-\mathrm{EA}(\mathrm{X})$ decreases along the $\mathrm{LiF}, \mathrm{LiCl}, \mathrm{NaF}$, and $\mathrm{NaCl}$ series, being 1.99, 1.77, 1.74 , and $1.52 \mathrm{eV}$, respectively. Thus, for a given external field, the crossing distance increases on going from the lightest halide to the heaviest, being very similar for $\mathrm{LiCl}$ and $\mathrm{NaF}$ (see column 3 of Table III for each molecule). The MS-CASPT2 computed values show this tendency and good agreement is found between these values and those derived using Eq. (2) applying the experimental data for EA and IP (cf. columns 1 and 3 of Table III) clearly indicating the excellent performance of MS-CASPT2 to accurately predict the crossing point. Notice that for $\mathrm{LiCl}$ and $\mathrm{NaF}$ the com- puted crossing distance at different electric fields is practically the same. This is a direct consequence of the MSCASPT2 values for IP(M)-EA(X), which are the same for both molecules, $1.85 \mathrm{eV}$. Even more remarkable is the excellent agreement observed for $\mathrm{NaCl}$ between the $R_{c}$ computed values and those derived from experimental data. Due to a cancellation of errors in the computation of $\operatorname{IP}(\mathrm{Na})$ and $\mathrm{EA}(\mathrm{Cl})$ the energy difference between both quantities, 1.53 $\mathrm{eV}$, essentially coincides with the experimental value. The largest errors in $\mathrm{IP}(\mathrm{M})-\mathrm{EA}(\mathrm{X})$ are found for the fluorides due to the well-known difficulty to accurately compute the $\mathrm{F}$ electron affinity. For LiF the computed value of $\operatorname{IP}(\mathrm{M})-$ $\mathrm{EA}(\mathrm{X}), 2.17 \mathrm{eV}$, is $0.18 \mathrm{eV}$ larger than the experimental value and therefore, for $\mathrm{LiF}$ the largest discrepancies between the MS-CASPT2 and empirical values, cf. Eq. (2), of $R_{c}$ are encountered. Finally, it is worth noting that when the strength of the electric field approaches the maximum value for which a crossing still occurs, the difference between the computed $R_{c}$ values and those derived from empirical data becomes larger. Near this limit, $R_{c}$ increases in an extremely rapid way with small variations of the intensity of the external field, and therefore, agreement with data derived from experiment is more difficult to reach. The maximum value of the electric field for which a crossing still takes place decreases on going along the $\mathrm{LiF}, \mathrm{LiCl}, \mathrm{NaF}$, and $\mathrm{NaCl}$ series. This is consistent with the prediction of Eq. (3), which shows that the maximum strength of the electric field is proportional to the IP $(\mathrm{M})-\mathrm{EA}(\mathrm{X})$ difference. The predicted values using experimental data and Eq. (3) are 1.34, 1.06, 1.02, and $0.78 \times 10^{-3}$ a.u. while those obtained applying Eq. (3) but using the computed values for $\mathrm{IP}(\mathrm{M})$ and $\mathrm{EA}(\mathrm{X})$ are 1.59, $1.16,1.16$, and $0.79 \times 10^{-3}$ a.u., for $\mathrm{LiF}, \mathrm{LiCl}, \mathrm{NaF}$, and $\mathrm{NaCl}$, respectively. As in the previous results, very good agreement between CASPT2 results and predictions made by the empirical Eq. (3) is found for $\mathrm{NaF}, \mathrm{LiCl}$, and $\mathrm{NaCl}$ and small differences for $\mathrm{LiF}$. 
TABLE IV. Diabatic coupling elements (in $\mathrm{meV}$ ) at the crossing point as a function of the applied homogeneous electric field $\left(10^{-3}\right.$ a.u. $)$.

\begin{tabular}{rrrrc}
\hline \hline Field & LiF & LiCl & NaF & $\mathrm{NaCl}$ \\
\hline-0.50 & 48.98 & 23.30 & 25.34 & 8.16 \\
0.00 & 39.45 & 8.95 & 9.75 & 3.49 \\
0.50 & 17.69 & 3.65 & 5.92 & 0.54 \\
0.75 & 14.97 & 1.91 & 4.48 & 0.06 \\
1.00 & 8.71 & 0.63 & $\mathrm{a}$ & \\
1.15 & 6.26 & 0.10 & 1.04 & \\
1.50 & 1.09 & & & \\
\hline \hline
\end{tabular}

${ }^{a}$ The two states are affected by intruder states. The use of a level shift technique does not solve the problem because of the small energy differences at the crossing point.

At this point, we turn our attention to the electronic coupling in the crossing region. The energy difference between the two adiabatic curves is a very small quantity and very sensitive to small changes in the $\mathrm{M}-\mathrm{X}$ internuclear distance and to the computational approach. From a simple approximation based on empirical parameters, it has been shown that the adiabatic splitting is an exponentially decreasing function of $R_{c},{ }^{14,33}$ vide infra. Therefore, a decrease of the energy difference between the two adiabatic curves at the crossing distance is expected on going from $\mathrm{LiF}$ to $\mathrm{LiCl}$, $\mathrm{NaF}$, and $\mathrm{NaCl}$. This is indeed the case; MS-CASPT2 diabatic coupling elements are found to be very small, with the largest value of $39.45 \mathrm{meV}$ for $\mathrm{LiF}$ and the smallest 3.49 $\mathrm{meV}$ for $\mathrm{NaCl}$. Very similar values are obtained for $\mathrm{LiCl}$ and $\mathrm{NaF}, 8.95$ and $9.75 \mathrm{meV}$, respectively, in accordance with the similar $R_{c}$ computed for both systems. These small coupling energy values result in very abrupt changes in the wave function around the crossing point. Indeed, the character of the wave function suddenly changes from ionic to covalent (or vice versa) in a short distance range, e.g., for $\mathrm{LiCl}$ the Mulliken charge for the cation in the lowest state changes in $0.5 \AA$ from 0.93 to 0.04 . When applying a positive uniform electric field along the internuclear direction, the molecule is perturbed, the crossing distance increases, and consequently, the diabatic coupling elements decrease. A summary of values for the electronic coupling element at the crossing point including the effect of a uniform external electric field is reported in Table IV. Contrarily to the case discussed above, a negative electric field, which destabilizes the ionic curve, leads to a shorter $R_{c}$ and a larger splitting. The clearly marked electric field effect on the crossing distance is the responsible for the increase observed in the electronic coupling term.

The discussion about the values of the diabatic electronic coupling above has been limited to the crossing point only. To investigate the variation of this parameter with the internuclear distance it is necessary to obtain a diabatic representation of the potential energy curves. For a two-state problem, the diabatic electronic coupling, $H_{12}$, is simply the nondiagonal element of the matrix representation of the Hamiltonian in the basis of the diabatic states. Several diabatization procedures have been described in the literature to obtain a suitable diabatic basis from the adiabatic states. Here, we quote the nearly diabatic method proposed by Cimiraglia et al. $^{34}$ based on second-order quasidegenerate perturbation theory, the empirical approach suggested by Kahn et $a l .{ }^{35}$ and the transformation proposed by Werner and Meyer ${ }^{13}$ based on the use of the matrix representation of the dipole moment. The first method requires the external definition of a reference and its application is rather involved. The second method assumes a Rittner potential for the ionic state and permits to obtain the diabatic coupling term in a direct way. Bauschlicher and Langhoff ${ }^{15}$ have chosen this method in their study of the neutral-ionic crossing in $\mathrm{LiF}$. However, it is not straightforward to extend this method to account for the presence of a uniform external electric field. Therefore, the method proposed by Werner and Meyer has been chosen in the present work.

For $\mathrm{LiF}$ in absence of any external electric field, the adiabatic and diabatic curves near the crossing region are reported in Fig. 2. These curves are representative of the rest of the molecules and, also, of the systems in presence of a

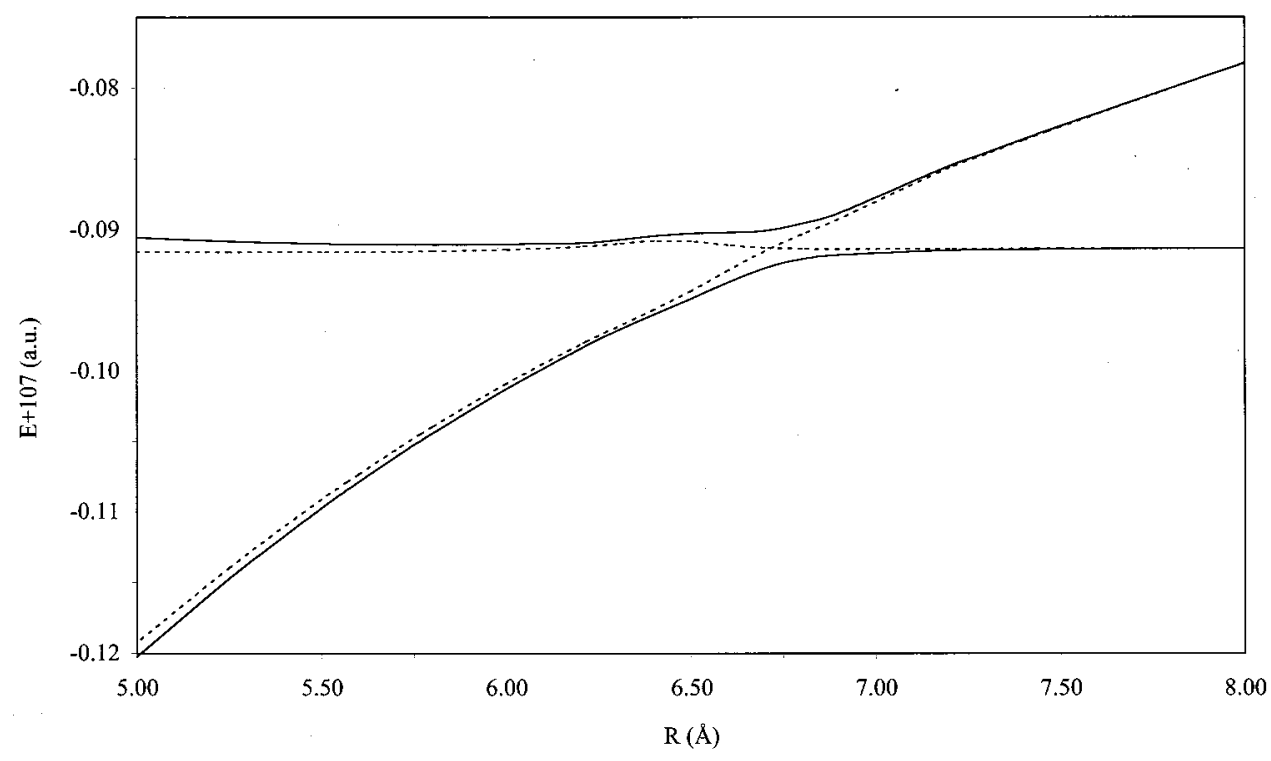

FIG. 2. Adiabatic and diabatic curves for $\mathrm{LiF}$ near the crossing region. 


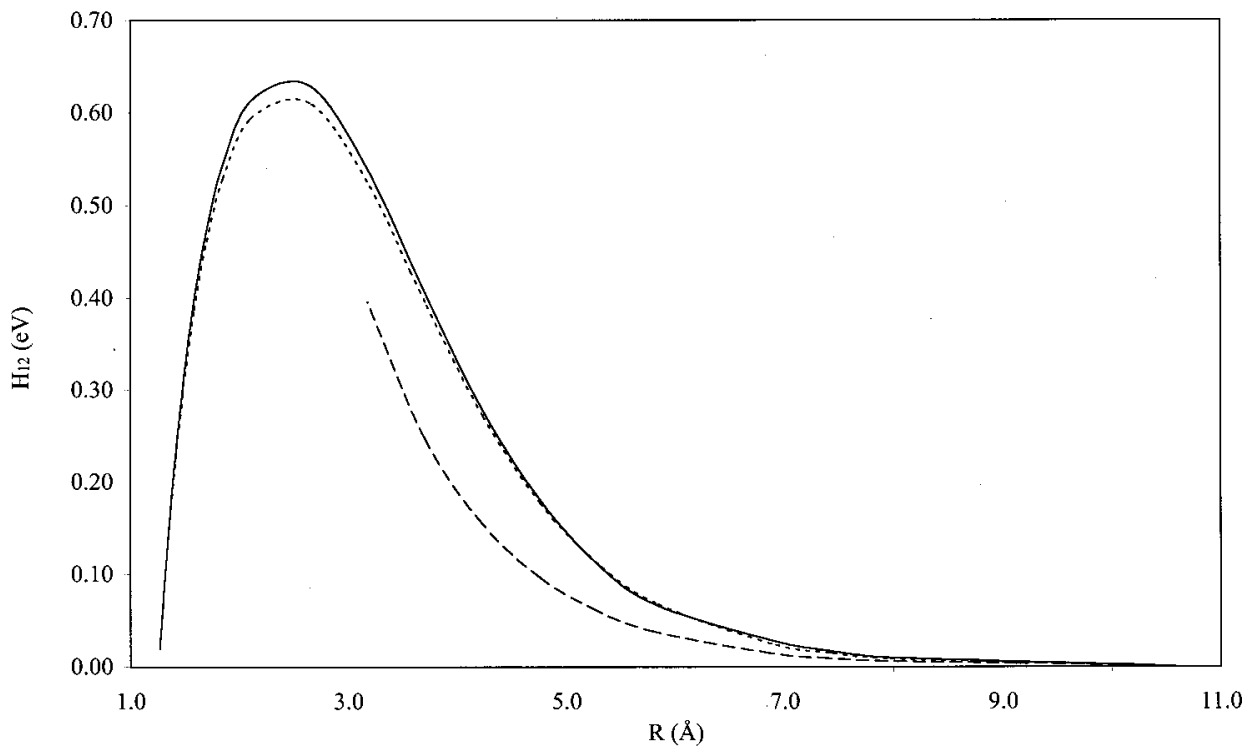

FIG. 3. Variation of the MS-CASPT2 diabatic coupling element of $\mathrm{LiF}$ with distance (solid line). Values from Werner and Meyer (Ref. 13) (dotted line) and from Grice and Herschbach (Ref. 14) (dashed line) are included for comparison. uniform external electric field. As expected from theory, $H_{12}\left(R_{c}\right)$ derived from the diabatic curves, $34.82 \mathrm{meV}$, is very close to that obtained taking half the energy difference of adiabatic potential energy curves, $39.45 \mathrm{meV}$. Furthermore, the behavior of $\mathrm{H}_{12}$ with respect to the internuclear distance is reported in Fig. 3, which for comparison purposes also includes the values reported by Werner and Meyer ${ }^{13}$ and those reported by Grice and Herschbach. ${ }^{14}$ This figure evidences again the satisfactory performance of MS-CASPT2 in the description of the neutral-ionic crossing. It is important to stress the fact that in presence of a uniform external electric field, the variation of $H_{12}$ with the distance is virtually the same that is obtained when the electric field is not present. Hence, the only changes in $H_{12}$ induced by the field arise from the change in the crossing distance. To summarize, MS-CASPT2 derived diabatic curves for $\mathrm{LiF}$ are in good agreement with previous accurate results. Hence, it is expected that the values obtained for $\mathrm{LiCl}, \mathrm{NaF}$, and $\mathrm{NaCl}$ (not explicitly reported) for which there is no previous information will be of similar accuracy.

Finally, it is important to point out that the diabatic curves described above can be appropriately scaled to reproduce the proper asymptotic energy. Different authors have previously employed this empirical scaling procedure. ${ }^{15,35}$ The main effect introduced by this scaling is to lower the ionic potential energy curve and, hence, to predict a larger value for $R_{c}$. For the molecules described in this work the largest effect is expected for $\mathrm{LiF}$, for which the largest errors in IP(M) and $\mathrm{EA}(\mathrm{X})$ are found. In fact, for this molecule and in absence of electric field, the scaling shifts $R_{c}$ from 6.78 to $7.31 \AA$ which is closer to the value predicted from the use of the Rittner potential, $7.24 \AA$. This variation in $R_{c}$ leads to a significant decrease in the $H_{12}$ value, from 39.45 to 18.95 $\mathrm{meV}$, in agreement with the dependence of $H_{12}$ on the distance depicted in Fig. 3. However, the $R_{c}$ value predicted by the MS-CASPT2 calculations for $\mathrm{NaCl}$ is very close to that obtained from Eq. (1) and, therefore, the scaling does not introduce any significant change in the value of the electronic coupling term for this molecule. Consequently, unless very accurate calculations are carried out to properly described $\mathrm{IP}(\mathrm{M})$ and $\mathrm{EA}(\mathrm{X})$ it is necessary to apply the scaling procedure to obtain precise values of the electronic coupling term. However, this scaling procedure will not change the qualitative conclusions reached in this work about the trends for the electronic coupling elements exhibited by the different molecules and for the effects induced by a uniform electric field.

\section{Relationship between the crossing distance and the ionic character of the bond}

Finally, we discuss a possible relationship between the crossing distance and the ionicity of the molecules. The calculated net Mulliken charges for the alkali atoms in the electronic ground state at the equilibrium distance are 0.99, 0.96, 0.87 , and 0.72 , for $\mathrm{NaF}, \mathrm{LiF}, \mathrm{NaCl}$, and $\mathrm{LiCl}$, respectively. Although Mulliken population analysis does not give a quantitative measure of the ionicity and have to be used with extreme care, ${ }^{36}$ it may be used to indicate a given trend. In fact, the ionicity order predicted by the Mulliken population analysis is in agreement with the Pauling ionicity scale. ${ }^{37}$ However, results in Table III show that the crossing distance along the series varies as $\mathrm{LiF}<\mathrm{LiCl} \approx \mathrm{NaF}<\mathrm{NaCl}$ and does not follow the trend in ionicity. As has been shown above, the EA and IP atomic parameters mainly determine the location of the crossing point. However, the ionic character of a given molecule does not only depend on these parameters, but also on the atomic polarizabilities. Despite the fact that the $\mathrm{Cl}$ EA is larger than that of $\mathrm{F}$, the larger polarizability of $\mathrm{Cl}^{-}$makes the alkali chlorides less ionic than the fluorides, the polarization of the anion electronic cloud towards the cation decreases the net charge in the anion atomic region. In addition, $\mathrm{Li}$ has a larger IP than $\mathrm{Na}$, but $\mathrm{Li}^{+}$polarizes its surrounding more efficiently than $\mathrm{Na}^{+}$, and, hence, the sodium halides are slightly more ionic than the litium halides. 


\section{CONCLUSIONS}

In this work the weakly avoided crossing between the two lowest ${ }^{1} \Sigma^{+}$electronic states of a series of alkali halide molecules has been studied by means of the recently reported MS-CASPT2 method. ${ }^{21}$ For a large enough basis set and a CASSCF that includes an important part of the radial and angular correlation of the outermost halide electrons, the calculated crossing distance is in fairly good agreement with that predicted from an empirical but physically wellgrounded model. The agreement between both approaches is related to the accurate description of the halogen EA and the metal IP obtained at this level of theory. Therefore, the MSCASPT2 method is found to be capable to adequately describe the avoided crossing region for a representative series of alkali halide molecules thus confirming recently reported previous results for $\mathrm{LiF}^{21}$

The study of the avoided crossing region on these molecules has been extended to include the effect of a uniform electric field and the empirical formula based on the Rittner potential has been generalized accordingly. Again, the predictions made by the MS-CASPT2 method are in agreement with those derived from the generalized Rittner potential. In particular, it is shown that the presence of an electric field that stabilizes the ionic asymptote enlarges the crossing distance and, decreases the diabatic coupling term. This is accompanied by a considerable decrease of the distance interval where the abrupt charge-transfer process takes place. The variation of the electronic coupling term with the internuclear distance has been obtained from the diabatic curves derived using the procedure proposed by Werner and Meyer. ${ }^{13}$ This method is also appropriate to study the crossing region in presence of a uniform external electric field. An important result emerging from the study of the diabatic curves obtained in the presence of a uniform external electric field is that the variation of $H_{12}$ with the distance follows the dependence obtained in absence of the external field. The main noticeable effect induced by the electric field being on the crossing distance only.

The ability of the MS-CASPT2 to properly describe the electronic structure at the avoided crossing region opens the possibility to quantitative studies of electron-transfer processes as those occurring at a surface, that are mediated by external electric fields or those involving rather complex biological systems. In particular, the quantitative ab initio quantum mechanical study of oxidation-reaction processes at surfaces seems to be at hand.

\section{ACKNOWLEDGMENTS}

Financial support has been provided by Spanish "Ministerio de Educación y Culturá" CICyT project PB98-1216CO2-01 and, in part, by "Generalitat de Catalunya" Grant No. 1999SGR-00040. Part of the computer time was provided by the CESCA/CEPBA supercomputer center thanks to a research grant from the University of Barcelona. C.deG. acknowledges the financial support through the TMR activity "Marie Curie research training grants" Grant No. FMBICT983279 established by the European Community and D.D. acknowledges the "Generalitat de Catalunya" for a predoctoral grant.

${ }^{1}$ K. P. Huber and G. Herzberg, Molecular Spectra and Molecular Structure, Vol. 4, Constants of Diatomic Molecules (van Nostrand-Reinhold, New York, 1979).

${ }^{2}$ F. Illas, J. M. Ricart, and M. Fernández-García, J. Chem. Phys. 104, 5647 (1996).

${ }^{3}$ F. Illas, J. Rubio, C. Sousa, A. Povill, S. Zurita, M. Fernández-García, J. M. Ricart, A. Clotet, and J. Casanovas, J. Mol. Struct.: THEOCHEM 371, 257 (1996).

${ }^{4}$ L. G. M. Pettersson and P. S. Bagus, Phys. Rev. Lett. 56, 500 (1986).

${ }^{5}$ P. S. Bagus, G. Pacchioni, and M. R. Philpott, J. Chem. Phys. 90, 4287 (1989).

${ }^{6}$ J. Rubio, J. M. Ricart, J. Casanovas, M. Blanco, and F. Illas, J. Electroanal. Chem. 359, 105 (1993).

${ }^{7}$ L. Langmuir and K. H. Kingdon, Phys. Rev. 21, 381 (1923).

${ }^{8}$ M. Scheffler, C. Droste, A. Fleszar, F. Maca, G. Wachutka, and G. Barzel, Physica B 172, 143 (1991).

${ }^{9}$ G. Pacchioni and P. S. Bagus, Surf. Sci. 286, 317 (1993).

${ }^{10}$ P. S. Bagus and G. Pacchioni, J. Chem. Phys. 102, 879 (1995).

${ }^{11}$ P. S. Bagus and F. Illas, Chem. Phys. Lett. 224, 576 (1994).

${ }^{12}$ E. S. Rittner, J. Chem. Phys. 19, 1030 (1951).

${ }^{13}$ H. J. Werner and W. Meyer, J. Chem. Phys. 74, 5802 (1981).

${ }^{14}$ R. Grice and D. R. Herschbach, Mol. Phys. 27, 159 (1974).

${ }^{15}$ C. W. Bauschlicher and S. R. Langhoff, J. Chem. Phys. 89, 4246 (1988).

${ }^{16}$ A. Zaitsevskii and J. P. Malrieu, Chem. Phys. Lett. 228, 458 (1994).

${ }^{17}$ J. P. Malrieu, J. L. Heully, and A. Zaitsevskii, Theor. Chim. Acta 90, 167 (1995).

${ }^{18}$ K. Andersson, P.-Å. Malmqvist, and B. O. Roos, J. Chem. Phys. 96, 1218 (1992).

${ }^{19}$ V. M. García, O. Castell, R. Caballol, and J. P. Malrieu, Chem. Phys. Lett. 238, 222 (1995)

${ }^{20}$ V. M. García, M. Reguero, R. Caballol, and J. P. Malrieu, Chem. Phys. Lett. 281, 161 (1997).

${ }^{21}$ J. Finley, P.-A. Malmqvist, B. O. Roos, and L. Serrano-Andrés, Chem. Phys. Lett. 288, 299 (1998).

${ }^{22}$ J. P. Finley and H. A. Witek, J. Chem. Phys. 112, 3958 (2000).

${ }^{23}$ H. Nakano, J. Chem. Phys. 99, 7983 (1993).

${ }^{24}$ M. J. Rosker, T. S. Rose, and A. H. Zewail, Chem. Phys. Lett. 146, 175 (1988).

${ }^{25}$ T. S. Rose, M. J. Rosker, and A. H. Zewail, J. Chem. Phys. 88, 6672 (1988).

${ }^{26}$ T. S. Rose, M. J. Rosker, and A. H. Zewail, J. Chem. Phys. 91, 7415 (1989).

${ }^{27}$ Y. Sakai, E. Miyoshi, and T. Anno, Can. J. Chem. 70, 309 (1992).

${ }^{28}$ K. Yamashita and K. Morokuma, Faraday Discuss. Chem. Soc. 91, 47 (1991).

${ }^{29}$ G. H. Peslherbe, R. Bianco, J. T. Hynes, and B. M. Ladanyi, J. Chem. Soc., Faraday Trans. 93, 977 (1997).

${ }^{30}$ K. Andersson, M. R. A. Blomberg, M. P. Fülscher, G. Karlström, R. Lindh, P.-A. Malmqvist, P. Neogrády, J. Olsen, B. O. Roos, A. J. Sadlej, M. Schütz, L. Seijo, L. Serrano-Andrés, P. E. M. Siegbahn, and P.-O. Widmark, MOLCAS Version 4, University of Lund, Lund, 1997.

${ }^{31}$ P.-O. Widmark, P.-A. Malmqvist, and B. O. Roos, Theor. Chim. Acta 77, 291 (1990).

${ }^{32}$ P.-O. Widmark, B. J. Persson, and B. O. Roos, Theor. Chim. Acta 79, 419 (1991).

${ }^{33}$ R. E. Olson, F. T. Smith, and E. Bauer, Appl. Opt. 10, 1848 (1971).

${ }^{34}$ R. Cimiraglia, J. P. Malrieu, M. Persico, and F. Spiegelmann, J. Phys. B 18, 3073 (1985).

${ }^{35}$ L. R. Kahn, P. J. Hay, and I. Shavitt, J. Chem. Phys. 61, 3530 (1974).

${ }^{36}$ P. S. Bagus, F. Illas, C. Sousa, and G. Pacchioni, in Electronic Properties of Solids Using Cluster Models, edited by T. A. Kaplan and S. D. Mahanti (Plenum, New York, 1995), p. 93.

${ }^{37}$ L. Pauling, The Nature of the Chemical Bond (Cornell University Press, Ithaca, NY, 1960). 